\title{
Association of Vitamin D Deficiency with Acute Lower Respiratory Infection in Toddlers
}

\author{
Narang GS ${ }^{1}$, Arora S $^{2}$, Kukreja S $^{3}$, Shifali ${ }^{4}$
}

${ }^{1}$ Professor Gursharn Singh Narang, MBBS, MD, ${ }^{2}$ Professor Sunita Arora, MBBS. MD Paediatrics, ${ }^{3}$ Professor Sahiba Kukreja, MBBS, MD Biochemistry, ${ }^{4} \mathrm{Dr}$. Shifali, MBBS, MD Resident. All from the Department of Paediatrics, Sri Guru Ram Das Institute of Medical Sciences and Research, Amritsar, Punjab, India.

\section{Address for correspondence:}

Dr. Gursharan Singh Narang, Professor

Department of Paediatrics

SGRDIMSR , Amritsar, Punjab, 143001

Tel: +91-9914233469

E-mail: gsnarang321@gmail.com

\section{Acknowledgments: None \\ Funding: Nil}

Conflict of Interest: This manuscript has been written as a part of the compulsory submission of the dissertation submitted to Baba Farid University of Health Sciences (BFUHS) in October 2015. This study was done from December 2013 till June 2015 in SGRDIMS\&R Amritsar.

Permission from IRB: Yes

Ethical dilemmas faced during study: None

\section{How to cite}

Narang GS, Arora S, Kukreja S, Shifali. Association of Vitamin D Deficiency with Acute Lower Respiratory Infection in Toddlers. J Nepal Paediatr Soc 2016;36(1):14-18

doi: http://dx.doi.org/10.3126/jnps.v36i1.13982

This work is licensed under a Creative Commons Attribution 3.0 License.

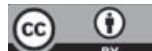

\begin{abstract}
Introduction: Pneumonia remains a significant health problem in India with significant morbidity and mortality. Objectives of this study were to identifying an effective nutritional agent that reduces the need for antibiotics, duration of pneumonia and length of hospitalization would be highly cost-beneficial. Material and Methods: This was a hospital-based casecontrol study: A total of 100 children including 50 cases and 50 controls, aged 2-60 months, were enrolled. Case definition of severe ALRI as given by the World Health Organization was used for cases. Controls were healthy children attending outpatients' service for immunization or admitted for minor ailments other than ALRTI. Results: Mean 25(OH)D concentrations in children of the study group were lower than those of the control group $(20.431 \mathrm{ng} / \mathrm{mL}$ vs. $27.67 \mathrm{ng} / \mathrm{mL} ; p=$ $0.035)$. Factors significantly associated with decreased risk of ALRTI in univariate analysis were: exclusive breastfeeding in the first 6 months (cases 13/50 (26\%), controls $42 / 50$ (84\%); $p=0.000)$; adequate exposure to sunlight (cases $14 / 50(28 \%)$, controls 42/50 (84\%); $\mathrm{P}=0.000)$; and serum $250 \mathrm{HD} 3<20 \mathrm{ng} / \mathrm{ml}$ (cases $28 / 50(56 \%)$, controls $0 / 50(0 \%) ; p=.000)$. Multivariate logistic model indicated that severe vitamin $D$ deficiency $(\mathrm{OR}=\mathrm{NA})$, exclusive breast feeding $(\mathrm{OR}=.174)$; $95 \% \mathrm{Cl}(.061$ $.521 ; p=.002)$ and adequate sunlight exposure (OR=.179) $; 95 \% \mathrm{Cl}(.062-.519) ; p=.002)$ were significant independent predictive risk factors for ALRTI among the children between 2 months -5 years of age (all $p<0.05$ ). Conclusion: Severe vitamin $D$ deficiency $(<20 \mathrm{ng} / \mathrm{ml})$, nonexclusive breastfeeding in the first six months of life, inadequate sun exposure were significant risk factors for ALRTI in Indian children.
\end{abstract}

Key words: ALRTI, pneumonia, vitamin D.

\section{Introduction}

- ach year, there are more than 150 million cases of childhood - pneumonia and nearly two million children under five lose their lives to an acute bout of pneumonia or another acute respiratory infection. Pneumonia remains a significant health problem in India with significant morbidity and mortality. There is increasing recognition 
that nutritional deficiencies, including micronutrients are important determinants of infections, including pneumonia, and their outcome. Identifying an effective nutritional agent that reduces the need for antibiotics, duration of pneumonia and length of hospitalization would be highly cost-beneficial. Clinical and subclinical vitamin $\mathrm{D}$ deficiency in children has been reported to be a significant risk factor for severe acute lower respiratory tract infection. Increased susceptibility to respiratory infections in vitamin $D$ deficiency may be explained on the basis of physical factors i.e. hypotonia and chest wall deformity, leading to reduced lung volume, poor compliance of the chest wall, atelectasis and fibrosis.

Apart from this, new knowledge of the biological and clinical importance of the active form of vitamin $D$ and its receptors has generated interest in its role in improving immune function. In Ethiopia researchers found that $42 \%$ of children in hospital with pneumonia had rickets, or severe vitamin $D$ deficiency ${ }^{1}$. Wayse- $V$ et al in a study found that subclinical vitamin D deficiency and nonexclusive breastfeeding in the first 4 months of life were significant risk factors for severe ALRI in Indian children ${ }^{2}$.Najada AS et al studied that rachitic infants are commonly hospitalized due to lower respiratory tract infections ${ }^{3}$. Karatekin $G$ et al found low vitamin D status to be associated with the risk of ALRI in Turkish neonates ${ }^{4}$. P.selvaraj et al studied that addition of $1,25(\mathrm{OH})_{2} \quad D_{3}$ might lead to increased expression of cathelicidin which could enhance the immunity against tuberculosis ${ }^{5}$.

The objectives of this study were to determine association of vitamin $D$ deficiency in children between 2 months to 5 years of age as a risk factor for acute lower respiratory tract infection (ALRTI).

\section{Material and Methods}

This was a hospital based case-control study was conducted in Sri Guru Ram Das Institute of Medical Sciences and Research Sri Amritsar, Punjab, India, in Dec 2013 to June 2015. Cases were children between 2 months to 5 years of age admitted as in-patients or seen in the outpatient department with ALRTI. A case definition of ALRTI as given by the World Health Organization (1995) was used for the cases: the presence of lower chest in drawing with respiratory rate more than 60 per minute in infants less than 2 months, more than 50 per minute in infants 3-12 months and more than 40 per minute in children 13-60 months. Controls were healthy children in same age group attending the clinic for vaccination during the study period or admitted for minor ailments other than ALRTI. Controls were not matched with cases.

A structured questionnaire was used to obtain information concerning age of the child; education of parents, recorded as illiterate, primary education, secondary, graduate or postgraduate; type of housing; smoking by any member in the family; and history of lower respiratory tract infection in family members. The child's immunization history was elicited from health records brought by parents. Breastfeeding history and the age of introduction of supplementary foods were recorded. The exclusive breastfeeding was categorized as $<6$ months or $>6$ months. Parents were explained the meaning of 'exclusive breastfeeding' (breast milk and nothing else) and asked if their child was exclusively breastfed during first 6 months of life. Dietary intake was assumed to be sufficient or insufficient based upon the caloric intake per day in pre morbid condition. Information regarding the practice of exposure of the infant to sunlight was collected including whether the infant was first exposed to outdoor sunlight before she/he learned to crawl, the frequency and approximate duration of the infant's sun exposure ( $<30 \mathrm{~min} />30 \mathrm{~min}$ per day) between $10 \mathrm{AM}$ to $2 \mathrm{PM}$, and how the infant was clothed when taken out into the sun. Adequate sun exposure was taken as spending $>30$ minutes per day in sunlight between 10 $\mathrm{AM}$ and $2 \mathrm{PM}$.

Weight was measured using an electronic scale. All the children who were enrolled had anthropometric parameters within normal limits for that particular age and gender. Data were analyzed using SPSS (Version 17.0). Standard statistical tests were used including $\mathrm{t}$ test, odds ratios and multiple logistic regression. For calculation of odds ratios, continuous variables were dichotomized: weight for age and height for age at Z-score, and age of introduction of dietary liquids or solids at the group median values. $\mathrm{P}<0.05$ was considered as statistically significant. $3 \mathrm{ml}$ of venous sample from study and control group children between 2 months - 5 years of age was drawn in two separate red topped vials (with clot activator), one for estimating vitamin $D$ and the other for serum ALP \& serum calcium. Care was taken not to apply the tourniquet while drawing the sample. These were sent to biochemistry lab of SGRDIMSR hospital after labeling the vials with patient's name and MRD number. After centrifugation, serum was separated and stored at -20 degree Celsius until analyzed. Vitamin D was estimated by Direct Elisa method, serum calcium by cresolphthalein complexone method and serum ALP by Pnnp (para nitro phenyl phosphate) method ${ }^{6}$. 


\section{Results}

It was seen that out of total 50 patients with ALRTI $28(56 \%)$ had severe vitamin D deficiency, $10(20 \%)$ had vitamin D deficiency and $12(24 \%)$ had sufficient vitamin $D$ levels. On the other hand in 50 children without ALRTI none of the cases had severe vitamin $D$ deficiency, $36(72 \%)$ had vitamin D deficiency and $14(28 \%)$ had sufficient levels. The difference was highly significant statistically $(p=0.000)$ thus indicating severe vitamin $D$ deficiency $(<20 \mathrm{ng} / \mathrm{ml})$ is an independent factor in increasing risk of ALRTI in pediatric age group. Mean [25(OH)D] was significantly lower among ALRTI cases than controls $(20.431 \mathrm{ng} / \mathrm{mL}$ vs. $27.67 \mathrm{ng} / \mathrm{mL} ; p=0.035)$.

Numbers of cases in group A (2- 24 months) were $35(70 \%)$, group B (25 - 36 months) were $10(20 \%)$, group C (37 - 48 months) were $4(8 \%)$, group D (4960 months) were $1(2 \%)$. Number of healthy controls in group A (2- 24 months) were 14(28\%), group B (25 - 36 months) were 15 (30\%), group C (37 - 48 months) were 11 (22\%), group D (49 - 60 months) were 10(20\%). Maximum number of cases of ALRTI were seen in age group A (2-24 months) $35(70 \%)$ followed by in age group B (25-36 months) 10(20\%). Distribution of healthy control children was almost equal in all 4 age groups. Mean age in study group was $\mathbf{2 0 . 8}$ months and $\mathbf{3 8 . 3 6}$ months in controls. Hence there was no statistically significant age predilection for risk of ALRTI. 13(26\%) cases and $42(84 \%)$ controls were exclusively breast fed in first 6 months of life.

On the other hand $37(74 \%)$ cases and $8(16 \%)$ controls were not exclusively breast fed in first 6 months of life. Statistical analysis revealed significant correlation of exclusive breast feeding with risk of acute lower respiratory tract infection with a $p$ value of 0.000 thus signifying role of exclusive breast feeding in first 6 months of life in prevention of ALRTI.

Total of $36(76 \%)$ cases and $8(16 \%)$ controls spent less than 30 minutes in sunlight .Only $14(28 \%)$ of the cases and $42(84 \%)$ controls spent $>30$ minutes.
Difference was statistically significant $(p=0.000)$. Thus inadequate sunlight exposure is directly related to risk of ALRTI .

Out of total 50 children with ALRTI 19(38\%) belong to lower class, $24(48 \%)$ belong to lower middle, $7(14 \%)$ belong to upper middle, and no case in upper class. On the other had among children without ALRTI 3(6\%) belonged to lower class, $16(32 \%)$ to lower middle, $25(50 \%)$ to upper middle, $6(12 \%)$ to upper class. So majority of children with ALRTI belonged to lower class and lower middle class. However majority of healthy children without ALRTI belonged to upper and lower middle class. Difference was statistically significant $(p=0.000)$ indicating the role of socioeconomic status in risk of ALRTI.

In the end variables showing statistically significant associations with ALRTI in univariate analyses were entered into multiple logistic regression. For calculation of adjusted odds ratio, continuous variables were dichotomized. $p<0.05$ was considered as statistically significant. Odds ratio was calculated and 95\% confidence interval were evaluated. In multiple logistic regression same factors were found significant for risk of ALRTI i.e, severe vitamin D deficiency, non exclusive breast feeding and inadequate sunlight exposure.

\section{Discussion}

Main finding of our study is that serum $25(\mathrm{OH}) \mathrm{D}$ concentrations in children between 2 months to 5 years of age with ALRTI (study group) were lower than those of the healthy children (control group) and most cases who had the serum $25(\mathrm{OH}) \mathrm{D}<20 \mathrm{ng} / \mathrm{ml}$ were more likely to develop $\operatorname{ALRTI}(P=.000)$. The study also showed that the mean $25(\mathrm{OH}) \mathrm{D}$ concentrations in children of the study group were lower than those of the control group (20.431 ng/mL vs. $27.67 \mathrm{ng} / \mathrm{mL} ; \mathrm{P}=0.035$ ). On the other hand there was no association of 25-hydroxy vitamin D $(25(\mathrm{OH}) \mathrm{D})$ deficiency with severity of acute lower respiratory infection (ALRI) in study group. In pediatric age group, various hospital based case-control studies

Table 1: Multivariate associations between ALRTI in children, vitamin d sufficiency, sunlight exposure, exclusive breast feeding and skin colour

\begin{tabular}{lcccc}
\hline Parameter & Cases $(\mathbf{N}=50)$ & Controls (N=50) & Odds Ratio(95\%(Cl) & p-value \\
\hline Sufficient Vitamin D & $12 / 50$ & $14 / 50$ & $0.387(.107-1.396)$ & 0.147 \\
\hline Severe Vitamin D Deficiency & $22 / 50$ & $0 / 50$ & $\mathrm{NA}$ & $\mathrm{NA}$ \\
\hline Exclusive Breast Feeding & $13 / 50$ & $42 / 50$ & $0.174(.061-.521)$ & 0.002 \\
\hline Sunlight Exposure >30 Min/Day & $14 / 50$ & $42 / 50$ & $0.179(.062-.519)$ & 0.002 \\
\hline Wheatish Skin Colour & $26 / 50$ & $26 / 50$ & $0.104(.021-.520)$ & 0.06 \\
\hline Frontal Bossing & $23 / 50$ & $27 / 50$ & $0.393(.123-1.258)$ & 0.116 \\
\hline
\end{tabular}


suggest that vitamin D deficiency may substantially increase the risk of pneumonia among children. Wayse $\underline{V}$ et al (2004) studied subclinical vitamin D deficiency in Indian children under 5 year of age as a significant risk factor for severe acute lower respiratory infection (ALRI). ${ }^{(2)}$ In Ethiopia, Muhe et al (1997) found that $42 \%$ of children in hospital with pneumonia had rickets, or severe vitamin $D$ deficiency $(<20 \mathrm{ng} / \mathrm{ml})$. $^{(1)}$

In our study significant correlation of exclusive breast feeding with risk of acute lower respiratory tract infection was seen $(P=.000)$. Supporting the results of our study, V Wayse et al (2004) found significant association of decreased risk of ALRTI with exclusive breast feeding in first 4 months and introduction of dietary liquids other than milk only after 6 months of life. (2) Broor s et al (2001) concluded lack of breast-feeding, upper respiratory infection in any family member, severe malnutrition, were significant risk factors associated with ALRTI in children. ${ }^{(7)}$ The protective effect of breastfeeding against severe ALRI is well recognized (World Health Organization Collaborative Study Team on the Role of Breastfeeding in the Prevention of Infant Mortality (WHO), 2000) ${ }^{8}$. In addition to this, in vitro studies (Rockett et al, 1998; Cantorna, 2000; Pichler et al, 2002) 9-11 $^{-1}$ and clinical studies of rickets (Muhe et al, $1997)^{1}$ support a role for vitamin D in protecting against infectious diseases.

In our study, inadequate sunlight exposure was found to be directly related to risk of ALRTI as well as levels of vitamin $\mathrm{D}$ deficiency in cases and controls. $36(72 \%)$ cases and $8(16 \%)$ controls had inadequate sunlight exposure from $10 \mathrm{AM}$ to $2 \mathrm{PM}$ i.e, spent less than 30 minutes in sunlight. Similar results were found in a study conducted by Ahmed P et al (2015). ALRTI was associated with less exposure to sunlight. Exposure to sunlight and vitamin $D$ supplementation contributed to vitamin $D$ status in this population. ${ }^{(14)}$ Wayse $v$ et al (2004) found if infant was not covered in heavy cloths when exposed to sunlight it was protective against ALRTI. ${ }^{(2)}$ Although India has abundant sunlight, low serum 250HD3 was common. Breast milk may not provide enough vitamin D for infants (World Health Organisation (WHO), 1998) ${ }^{12}$, especially if the mothers are also vitamin D-deficient (Andran et al, 2002) ${ }^{13}$.

\section{Conclusion}

Multivariate logistic model indicated that severe vitamin D deficiency, non- exclusive breast feeding and inadequate sunlight exposure were significant independent risk factors for ALRTI. But there was no association of vitamin $D$ deficiency with severity of pneumonia. Exposure to sunlight $>30 \mathrm{~min}$ a day was found to be associated with reduced risk of ALRI likely via vitamin $D$ synthesis mechanism. In addition to this, level of vitamin $D$ deficiency was inversely related to duration of sunlight exposure in both cases and controls. However association of vitamin D deficiency according to vitamin D supplementation could not be studied as no child in our study was getting vitamin $\mathrm{D}$ supplements.

\section{References}

1. Muhe $L$ et al. Case-control study of the role of nutritional rickets in the risk of developing pneumonia in Ethiopian children. The Lancet 1997; 349:1801-4.

2. Wayse $\mathrm{V}$ et al. Association of subclinical vitamin $D$ deficiency with severe acute lower respiratory infection in Indian children under 5 y. Eur $\mathrm{J}$ Clin Nut 2004;58(4):563-7.

3. Najada AS, Habashneh MS, Khader M. The frequency of nutritional rickets among hospitalized infants and its relation to respiratory diseases. $J$ Trop Pediatr 2004;50(6):364-8.

4. Karatekin $\mathrm{G}$ et al. Association of subclinical vitamin D deficiency in newborns with acute lower respiratory infection and their mothers. Eur $J$ of Clin Nut 2009;63(4):473-7.

5. Selvaraj $P$, Prabhu Anand $S$, Harishankar M, Alagarasu K. Plasma 1,25 Dihydroxy Vitamin $\mathrm{D}_{3}$ Level and Expression of Vitamin D Receptor

and Cathelicidin in Pulmonary Tuberculosis. J Clin Immunol 2009;29(4):470-8.

6. Holock MF .Vitamin D status :Measurement, Interpretation and Clinical Application. Ann Epidemiol 2009;19(2):73-8.

7. S. Broor, R.M. Pandey, M. Ghosh, R.S. Maitreyi, Rakesh Lodha, Tanu Singhal and S.K. Kabra risk factors for severe acute lower respiratory tract infection in under five children. Pediatrics 2001;38:1361-9

8. World Health Organization Collaborative Study Team on the Role of Breastfeeding in the Prevention of Infant Mortality; Effect of breastfeeding on infant and child mortality due to infectious diseases in less developed countries: a pooled analysis. Lancet 2000;355:451-5.

9. Rockett KA, Brookes R, Udalova I, Vidal V, Hill AVS, Kwiatkowski D. 1,25- Dihydroxyvitamin D3 induces 
nitric oxide synthase and suppresses growth of Mycobacterium tuberculosis in a humanmacrophagelike cell line. Infect Immun 1998;66: 5314-21.

10. Cantorna MT (2000): Vitamin D and autoimmunity: is vitamin $D$ status an environmental factor affecting autoimmune disease prevalence? Proc Soc Exp Biol Med 2000;223:230-3.

11. Pichler J, Gerstmayr M, Szepfalusi Z, Urbanek R, Peterlik M, Willheim M. 1a,25(OH)2D3 inhibits not only Th1 but also Th2 differentiation in human cord blood T cells. Pediatrics 2002;52:12-8.
12. World Health Organization (1998): Complementary Feeding Of Young Children In Developing Countries. Geneva: WHO.

13. Andran N, Yordam N, Ozon A: Risk factors for vitamin $D$ deficiency in breast-fed newborns and their mothers. Nutrition 2002;18:47-50.

14. Ahmed P, Babaniyi IB, Yusuf KK, Dodd C, Langdon G, Steinhoff M, Dawodu A. Vitamin D status and hospitalisation for childhood acute lower respiratory tract infections in Nigeria. Paediatr Int Child Health 2015;35(2): 151-6. 